\title{
Reduction of Electric and Magnetic Field Emissions caused by Overhead Power Lines
}

\author{
K. Deželak ${ }^{1}$, G. Štumberger ${ }^{1}$ and F. Jakl ${ }^{1}$ \\ ${ }^{1}$ Universty of Maribor \\ Faculty of Electrical Engineering and Computer Science \\ Smetanova 17, 2000 Maribor, Slovenia \\ Phone/Fax number:+3862220 7180 / +3862220 7272, e-mail: klemen.dezelak@uni-mb.si
}

\begin{abstract}
Calculations and measurements of magnetic and electric fields of overhead power lines existing in Slovenia show that electromagnetic field emissions on the border of overhead power line right of way could be too high. The allowed electromagnetic field emissions defined by Slovenian government in 1996 are very strict especially for newly constructed overhead lines. For that reason some modification should be applied for newly constructed overhead lines. This paper proposed a few solution of aforementioned problem regarding arrangements of overhead power line conductor.
\end{abstract}

\section{Key words}

Overhead lines, magnetic field, electric field, conductor arrangements.

\section{Introduction}

Nowadays different aspects especially those related with environmental impact have very important role. In the same sense the constructions of new overhead power lines or even reconstruction of old power lines require consideration of those aspects. For all that the most important role has a government legislation accepted by individual country. In case of electric and magnetic field emissions the European Union recommends some values, while each individual country accepts their own limit values. In 1996 Slovenia accepted quite strict values of magnetic and electric field emissions on the borders of overhead power lines right of way [1]. Those values are especially strict for newly constructed or only reconstructed overhead lines $(B=10 \mu \mathrm{T}, E=0.5 \mathrm{kV} / \mathrm{m})$, while for old, existent overhead lines limits values are not so strict. For that reason, old practises of overhead lines constructions can not be applied for newly constructed overhead lines. This work deals with possible solutions, which can be applied in the process of the new overhead line construction, to reduce electromagnetic fields emissions [2], [3]. Solutions can be divided into two classes. The first class deals with conductor rearrangements, while the second class is considered by passive circuits. This work deals only with the different applications of the first class, related to the overhead power line conductor re-arrangements. They are presented in the section 3 in form of three different solutions.

\section{Calculations of electric and magnetic field emissions}

Figs. 1 and 2 show typical Slovenian single-circuit and double-circuit $400 \mathrm{kV}$ overhead power line towers with two conductor bundle.

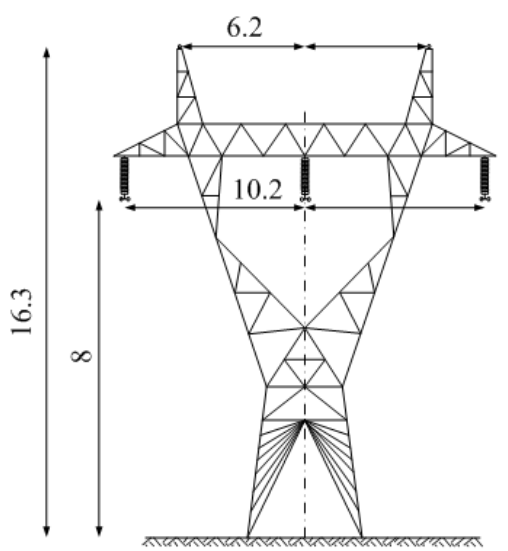

Fig. 1. Typical Slovenian single-circuit overhead power line tower.

The electric field caused by the charge distribution $q$ on the overhead lines can be calculated by (1), where $\alpha_{1}$ and $\alpha_{2}$ are angles between the beginning and the ending point of the conductor element $d \mathbf{l}$ (with the length $d l$ ) and the point of observation (Fig. 3). Vector a, with the length $a$, stands for the vector orthogonal to the $d \mathbf{l}$, which points from the direction of $d \mathbf{l}$ to an arbitrary point of observation. In the similar way the calculation of magnetic field caused by the current $i$ can be obtained by (2). 


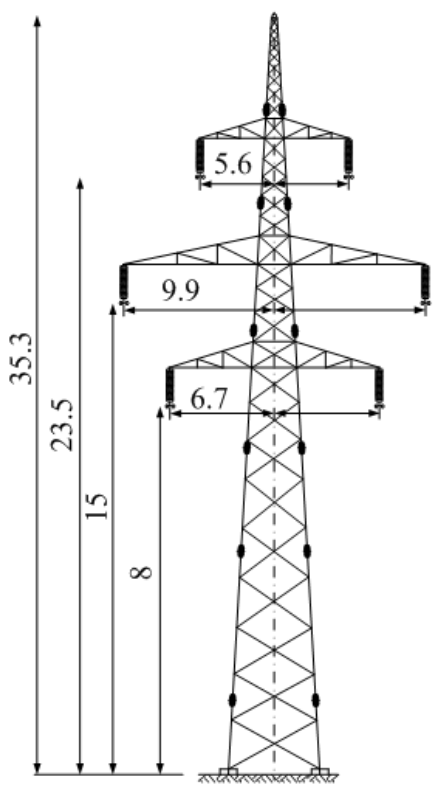

Fig. 2. Typical Slovenian double-circuit overhead power line tower.

$$
d \mathbf{E}=\frac{q}{4 \pi \varepsilon_{0} a}\left(\left(\sin \alpha_{2}-\sin \alpha_{1}\right) \frac{d \mathbf{l}}{d l}+\left(\cos \alpha_{1}-\cos \alpha_{2}\right) \frac{\mathbf{a}}{a}\right)
$$

$$
d \mathbf{B}=\frac{\mu_{0} i}{4 \pi a}\left(\cos \alpha_{1}-\cos \alpha_{2}\right)\left(\frac{d \mathbf{l}}{d l} \times \frac{\mathbf{a}}{a}\right)
$$

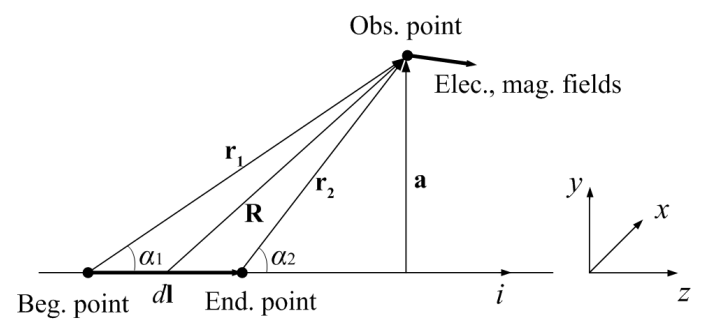

Fig. 3. The conductor element.

Results of magnetic and electric field calculations for Slovenian overhead lines are shown on Figs. 4 and 5, where $x$ is the axis along the overhead line right of way. As Figs. 4 and 5 show, the magnetic $(B)$ and electric $(E)$ field on the border of overhead power line right of way $(x$ $= \pm 25 \mathrm{~m})$ are higher that prescribed limits. For that reason some arrangements should be made. Values on Figs. 4 and 5 are root mean square (rms) obtained by integration of $B$ and $E$ vectors over the whole period $T=$ $0.02 \mathrm{~s}$ (for frequency $50 \mathrm{~Hz}$ ).

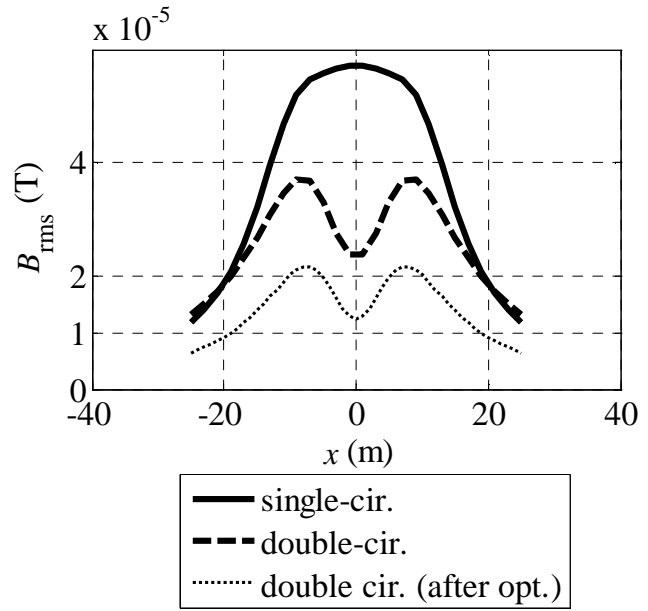

Fig. 4. Magnetic field density calculations for single-circuit and double-circuit Slovenian overhead power lines.

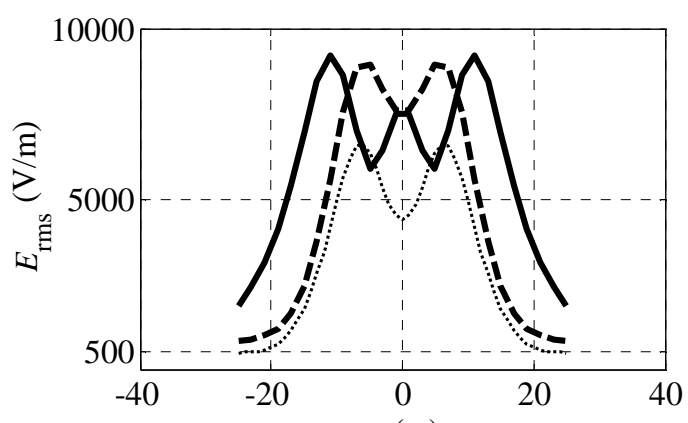

$x(\mathrm{~m})$

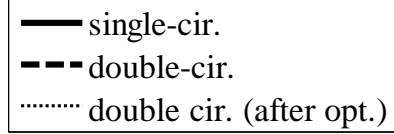

Fig. 5. Electric field strength calculations for single-circuit and double-circuit Slovenian overhead power lines.

\section{Reduction of electric and magnetic field emissions}

This work focuses on reduction of electromagnetic field emissions regarding to the overhead power line conductor re-arrangements [4]. The first solution, possible only for double-circuit overhead lines, is exchanging of conductors succession [5], [6]. The second solution is the usage of higher overhead lines tower, where conductor arrangements is the same, while the third solution is reduction of electromagnetic field emissions based on conductor arrangements obtained in an optimization process [7], [8].

Basically all three solutions can be classified into the rearrangement of the phase conductor class [4]. It is well known that electric and magnetic fields are substantially dependent on the line geometry, clearances between individual conductors and clearances between individual phases. That means that both, electric and magnetic, field emissions can be varied by the conductor position changing or by the conductor succession changing.

According to the Slovenian decree [1] the magnetic and electric field emissions should not exceed the prescribed limit values on the border of overhead power line right of way. The width of the aforementioned right of way is \pm 
$25 \mathrm{~m}$ from the axis along the overhead line. The height of the observed point is $1 \mathrm{~m}$ above earth.

Firstly the reduction of electric and magnetic field emissions caused by conductor succession changing was handled. This method can be applied only for doublecircuit overhead power lines (Fig. 2). For the left circuit the conductor succession A was used, while for the right circuit the six different conductor successions (a, b, c, d, e and f) were applied (Fig. 6). In Fig. 6 the number 1 stands for the first phase, the number 2 stands for the second phase and the number 3 stands for the third phase.

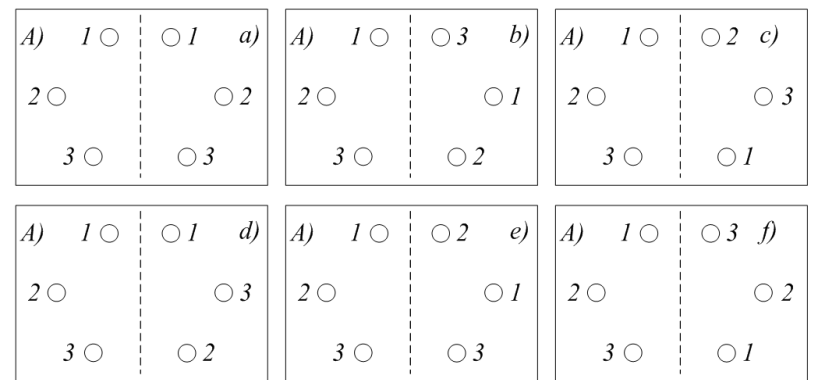

Fig. 6. Different conductor successions of right circuit applied on the double-circuit overhead line.

Results obtained for all six different combinations are shown on Figs. 7 and 8 and in Tab.1.
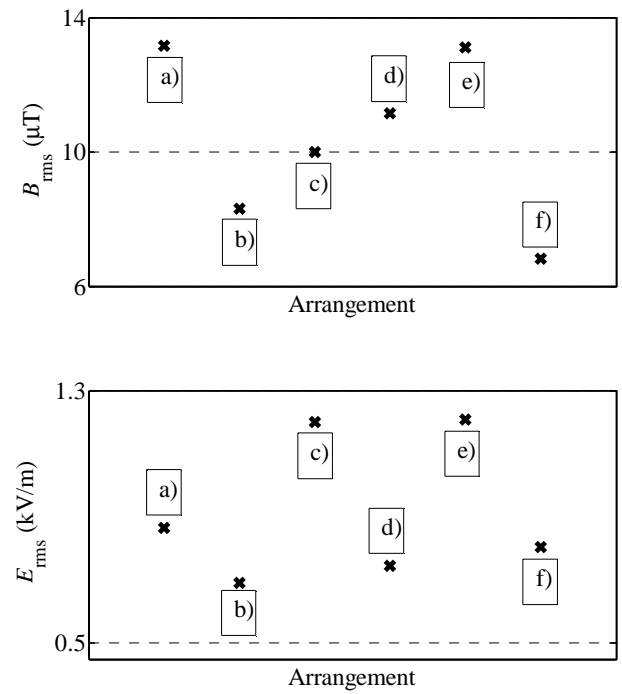

Fig. 7. Electric field strength and magnetic field density calculations (- $25 \mathrm{~m})$ for double-circuit overhead power lines.
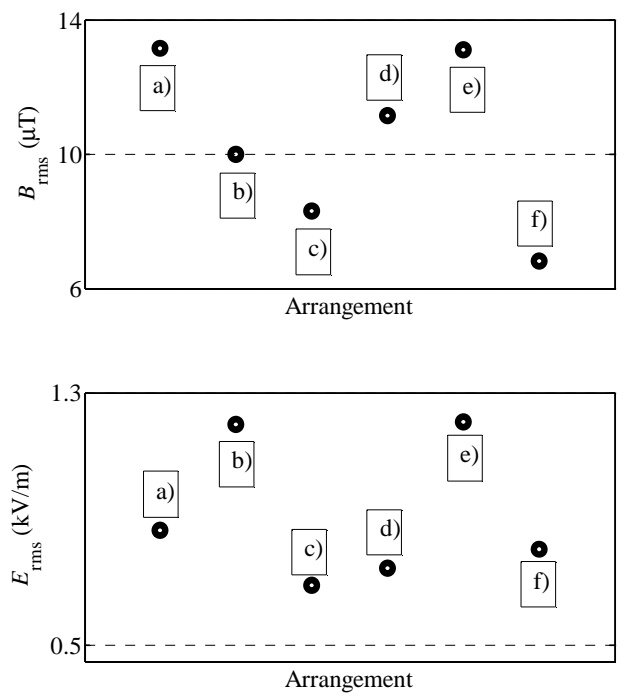

Fig. 8. Electric field strength and magnetic field density calculations $(+25 \mathrm{~m})$ for double-circuit overhead power lines

Tab. 1. Calculated electric and magnetic field emissions on the border of the overhead double-circuit line right of way.

\begin{tabular}{|l|l|l|l|l|}
\hline \multirow{2}{*}{ Arrang. } & \multicolumn{2}{|c|}{$B_{\mathrm{rms}}[\mu \mathrm{T}]$} & \multicolumn{2}{c|}{$E_{\mathrm{rms}}[\mathrm{kV} / \mathrm{m}]$} \\
\cline { 2 - 5 } & $-25[\mathrm{~m}]$ & $+25[\mathrm{~m}]$ & $-25[\mathrm{~m}]$ & $+25[\mathrm{~m}]$ \\
\hline a) & 13.16 & 13.16 & 0.864 & 0.864 \\
\hline b) & 8.31 & 10.00 & 0.691 & 1.199 \\
\hline c) & 10.00 & 8.31 & 1.199 & 0.691 \\
\hline d) & 11.15 & 11.15 & 0.746 & 0.746 \\
\hline e) & 13.10 & 13.10 & 1.207 & 1.207 \\
\hline f) & 6.82 & 6.82 & 0.805 & 0.805 \\
\hline
\end{tabular}

All combinations can be evaluated by the norm $N$ (3), where $\mathbf{x}(4)$ is the vector composed of the root mean square values of electric and magnetic field $\left(E_{\mathrm{rms}}, B_{\mathrm{rms}}\right)$ on the border of overhead line right of way $( \pm 25 \mathrm{~m})$. Combination with the lowest norm value $N$ can be handled as the best possible combination.

$$
\begin{gathered}
N=\frac{1}{4}\left(\mathbf{x} \cdot \mathbf{x}^{\mathrm{T}}\right) \\
\mathbf{x}=\left[\begin{array}{llll}
B_{\mathrm{rms},-25} & B_{\mathrm{rms},+25} & E_{\mathrm{rms},-25} & E_{\mathrm{rms},+25}
\end{array}\right]
\end{gathered}
$$

From that reason from all different conductor successions combinations, the combination $\mathrm{f}$ ) can be treated as the best one.

The second solution for reducing electric and magnetic field applied in this work is usage of higher towers. That means that the distance from point of interest to the overhead lines becomes larger. First proposed solution of this work can be applied only for double-circuit overhead lines, while this solution can be applied as well for single-circuit lines (Fig. 9). 

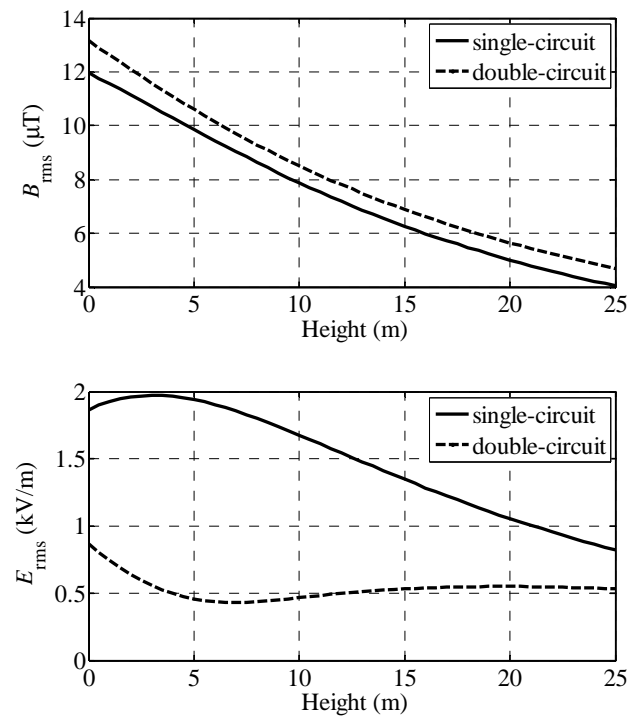

Fig. 9. $E_{\mathrm{rms}}$ and $B_{\mathrm{rms}}$ calculations for the single-circuit and the double-circuit Slovenian overhead power lines dependent on the increased height.

The height $0 \mathrm{~m}$ on Fig. 9 means that the height from Figs. 1 and 2 is valid, while the height of overhead lines $25 \mathrm{~m}$ means that all distances from Figs. 1 and 2 are higher for $25 \mathrm{~m}$ - the height of lower conductors is $33 \mathrm{~m}$.

As Fig. 9 shows the rms values of magnetic field ( $\left.B_{\mathrm{rms}}\right)$ becomes lower for higher heights as well for singlecircuit as for double-circuit.

On the other hand the calculated results for rms electric field strength $\left(E_{\mathrm{rms}}\right)$ on the border of overhead power line right of way are much more complex. For the singlecircuit line the $E_{\mathrm{rms}}$ with higher tower is firstly increasing, but after $3 \mathrm{~m}$ the $E_{\mathrm{rms}}$ value starts to decrease. On the other hand electric field of the double-circuit lines is firstly decreasing up to $7 \mathrm{~m}$ height. After that the $E_{\mathrm{rms}}$ value is slowly increasing and at approximately at $20 \mathrm{~m}$ height starts to decrease again.

The first treating solution for reducing $B$ and $E$ caused by overhead lines shows that $B$ and $E$ can be reduced (Tab. 1 ), but values of $E$ is still higher than prescribed. The second solution shows that with higher tower the magnetic and electric field can be reduce but due to essential increase of costs this solution is not appropriate. For that reason the third solution of reducing $B$ and $E$ based on conductor arrangements obtained by an optimization process was applied. The basic idea is that each conductor position is described by two parameters changing during optimization, while main parameters of objective function are magnetic and electric field on the order of overhead power line right of way. The applied optimization method is Differential Evolution [9].

Examples of magnetic and electric field values calculated for double-circuit overhead line with the conductor arrangements obtained in an optimization process [7], [8] are shown on Figs. 4 and 5. The root mean square values of magnetic and electric field obtained by third proposed solution do not exceed prescribed limit values [1].

\section{Conclusion}

This work deals with three possible solutions for reducing electric and magnetic field emissions on the border of overhead power line right of way. The first solution is in exchanging of conductor phase succession, the second is application of higher towers and the third in conductor re-arrangements obtained by an optimization process. In the third case the electric and magnetic field emissions can be reduced even with the lower towers in comparison with the existing overhead power line towers [7], [8].

\section{References}

[1] Official Gazette of the Republic of Slovenia No. 70/96, "The Decree on Electromagnetic Radiation in the Natural and Living Environment”, 1996.

[2] A. R. Panicali, "Calculation of Electric Fields due to Lines of Charge", IEEE Transactions on Electromagnetic Compatibility, 1991, 33, (1), pp. $67-68$.

[3] W. T. Kaune, L. E. Zaffanella, "Analysis of Magnetic Fields Produced Far from Electric Power Lines", IEEE Transactions on Power Delivery, 1992, 7, (4), pp. 2082 - 2091.

[4] R. Conti, A. Giorgi, R. Rendina, L. Sartore, E. A. Sena, "Technical Solutions to Reduce $50 \mathrm{~Hz}$ Magnetic Fields from Power Lines", 2003, June, IEEE PowerTech Conference, Bologna, Italy.

[5] I. Said, H. B. Hussain, "Computation of magnetic field from quadruple tower transmission lines in Malaysia", Universities Power Engineering Conference, 2008, UPEC 43rd, pp. 1 - 5.

[6] E. I. Mimos, D. K. Tsanakas, A. E. Tzinevrakis, "Solutions for high voltage transmission in suburban regions regarding the electric and magnetic fields", 2008, Automation Conqress, WAC, pp. $1-6$.

[7] K. Deželak, G. Štumberger, F. Jakl, "Overhead Power Line Emissions of Electromagnetic field Reduced by Optimization", BHK CIGRE, Neum, 2009.

[8] K. Deželak, G. Štumberger, F. Jakl, "Arrangements of overhead power line conductors determined by differential evolution", HRO CIGRE, Cavtat, 2009.

[9] R. M. Storn, K. V. Price, "Minimizing the Real Functions of the ICEC'96 Contest by Differential Evolution", IEEE Conference on Evolutionary Computation, Nagoya, Japan, 1996, pp. 842-844. 